\title{
ARTICLE
}

\section{INCL Intra-Nuclear Cascade and ABLA De-Excitation Models in Geant4}

\author{
Pekka KAITANIEMI ${ }^{1,3, *}$, Alain BOUDARD ${ }^{1}$, Sylvie LERAY ${ }^{1}$, Joseph CUGNON $^{2}$ and Davide MANCUSI ${ }^{2}$ \\ On behalf of the Geant4 collaboration \\ ${ }^{1}$ CEA/Saclay, DSM/IRFU/SPhN, 91191 Gif-sur-Yvette Cedex, France \\ ${ }^{2}$ University of Liége, Allée du 6 Août 17, bât B5, B-4000 Liége, Belgium \\ ${ }^{3}$ Helsinki Institute of Physics, P.O.Box 64 (Gustaf Hällström street 2), FIN-00014 University of Helsinki, Finland
}

\begin{abstract}
We present INCL intra-nuclear cascade and ABLA de-excitation (evaporation/fission) models that can be used to calculate collisions between projectile particles and nuclei at energies from 0.2 to $3 \mathrm{GeV}$. Supported projectiles are proton, neutron, pions and light ions up to alpha. The optimal target materials range from carbon to uranium.

We have extended the INCL model light ion projectile support up to carbon-ions and compared the results against thin and thick target data and Geant4 Binary cascade model. Both thin and first thick target results of the INCL model are in good agreement with experimental data.
\end{abstract}

KEYWORDS: Geant4, intra-nuclear cascade, Monte Carlo, ion-ion collisions

\section{Introduction}

INCL $^{1)}$ intra-nuclear cascade model calculates the reactions of nucleon, pion or light ion projectiles with target nuclei. After the cascade an excited remnant nucleus is de-excited using the $\mathrm{ABLA}^{2-4)}$ evaporation/fission code. INCL/ABLA alone is a thin-target simulation code. In order to perform calculations that involve complex geometries and multiple composite materials INCL must be embedded into a transport code that can offer this functionality. Two such transport codes are Geant $4^{5)}$ and MCNPX. ${ }^{6,7)}$ The original FORTRAN version of INCL4.2 has been included in MCNPX. ${ }^{8)}$ The same version of INCL was later translated to $\mathrm{C}++$ and included in Geant $4 .^{9)}$

The combination of INCL/ABLA with transport code has opened new possibilities for practical applications. Some of the more traditional applications for intra-nuclear cascade models are nuclear waste transmutation and Accelerator Driven Systems (ADS) studies which mainly deal with heavy target materials and nucleon beams. In recent years there has also been an increasing interest in medical applications of these Monte Carlo codes. One such application is the treatment of tumors using light ions, especially carbon beams with energies of a few hundred $\mathrm{MeV}$ per nucleon. Another interesting application for ion-ion collisions are spacecraft radiation damage and shielding studies. In this case the relevant ions range from protons to iron ions with energies up to a few $\mathrm{GeV}$.

This has motivated us to extend the light ion projectiles supported by INCL. In the previous release of INCL4.2 we supported light ion projectiles up to alpha. In this new version we have extended this support, in a fairly crude way, up to

\footnotetext{
*Corresponding author, E-mail:pekka.kaitaniemi@gmail.com
}

carbon. In this paper we present our first results and compare against Geant4 Binary cascade which offers similar support for light ion projectiles as INCL.

In the future we hope to further extend the ion-ion support of the INCL intra-nuclear cascade model and make it more realistic. This, and many technical shortcomings of the INCL codebase that have their roots in the FORTRAN77 legacy of the original code, have motivated us to perform a complete redesign of the INCL simulation code in object oriented $\mathrm{C}++$. We will discuss some of our motivations and main ideas of the redesign work and how they will help us to develop our model further.

\section{INCL4.2 Light Ion Extension}

The INCL light ion extension consists of two main parts: handling of the projectile as a collection of individual nucleons and de-excitation of the projectile fragments after the reaction. The main cascade in the target nucleus is treated following the standard INCL cascade procedure as described in Reference 1.

\section{Projectile as a Collection of Nucleons}

The projectile is modelled as a collection of independent nucleons with gaussian momentum and position distributions. ${ }^{10)}$ For the position distribution of the projectile nucleons we use the realistic standard deviation of the projectile ion. The standard deviation of the momentum distribution is fixed to $100 \mathrm{MeV} / \mathrm{c}$. When generating momentum distribution for the projectile nucleons we first generate it in the rest frame of the projectile and then boost it to the laboratory frame. The sum of the energies of the projectile nucleons is equal to the total energy of the projectile. The momentum of 

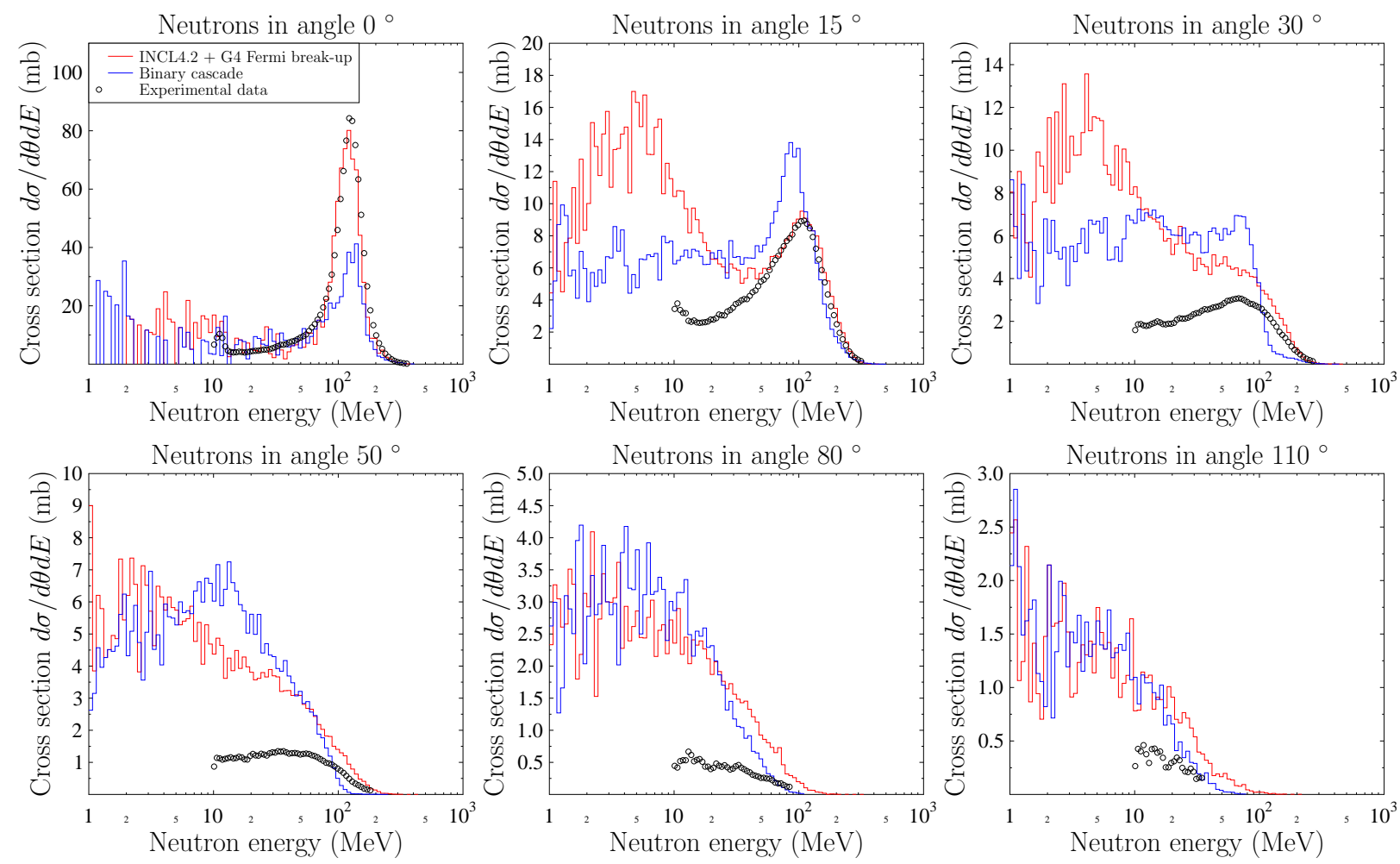

Fig. 1 Double-differential neutron energy spectra for $\mathrm{C}+\mathrm{C}$ at $135 \mathrm{MeV} /$ nucleon. INCL4.2 with Geant4 Fermi break-up and Geant4 Binary cascade are compared against experimental data from Reference 12.

the projectile nucleons is slightly rescaled due to the fact that they are on mass shell $\left(E^{2}-p^{2}=m^{2}\right)$ which is not compatible with the conservation of energy and momentum due to the binding energy of the projectile nucleus.

Some projectile nucleons miss the target. These are considered projectile spectators. Some of the nucleons that enter the target nucleus can pass through it without any collisions at all and are also considered as projectile spectators. The rest of the projectile nucleons proceed to produce an intra-nuclear cascade in the target as described in Reference 1.

At the end of the cascade all projectile spectators are combined into a spectator nucleus. The mass, charge and momentum of this nucleus are determined by direct summation on individual nucleons. The excitation energy is determined as follows. In the initialization of the cascade, a list of ion-projectile Fermi momenta has been randomly chosen corresponding to individual kinetic energies of the projectile nucleons. The removal of some projectile nucleons by the interaction with the target nucleus is interpreted as holes in the projectile spectator nucleus. Its excitation energy is simply the energy released in packing the spectator nucleons in the lowest energy states of the individual kinetic energy list.

\section{De-Excitation}

The spectator nucleus is always de-excited using the Geant4 Fermi break-up model. The target remnants are treated differently according to their mass at the end of the cascade: remnant nuclei lighter than mass 17 are de-excited with Geant4 Fermi break-up model and heavier ones with the ABLA fission/evaporation code. The ABLA version in Geant 4 provides us with evaporation of protons, neutrons and alphas that competes with fission channel for sufficiently heavy remnant nuclei.

\section{Results}

We have compared the new version of INCL against experimental data and the Geant 4 Binary cascade. These calculations have been done using a test version of INCL and Geant4 version geant 4-09-03-ref-05 which is an internal Geant 4 development release from May of 2010.

\section{Thin Target Calculations}

Double-differential energy spectra for neutrons produced in reaction $\mathrm{C}+\mathrm{C}$ at $135 \mathrm{MeV} /$ nucleon are shown in Fig. 1 . Agreement of the INCL4.2 model with experiment is very good in the forward region. INCL combined with Geant4 Fermi break-up reproduces the quasi-elastic peak very well, as can be seen in the energy spectrum for angle $0^{\circ}$. The agreement with experimental data is worse for angles above $30^{\circ}$.

In the case of $\mathrm{C}+\mathrm{C}$ at $290 \mathrm{MeV} /$ nucleon (Fig. 2) INCL performance is still fairly good, but not quite perfect, in the forward region. Both models show fairly similar levels of agreement with experiment. Overall INCL seems to reproduce the shape of the distribution slightly better than Binary cascade.

We have also compared oxygen and chlorine fragmentation 

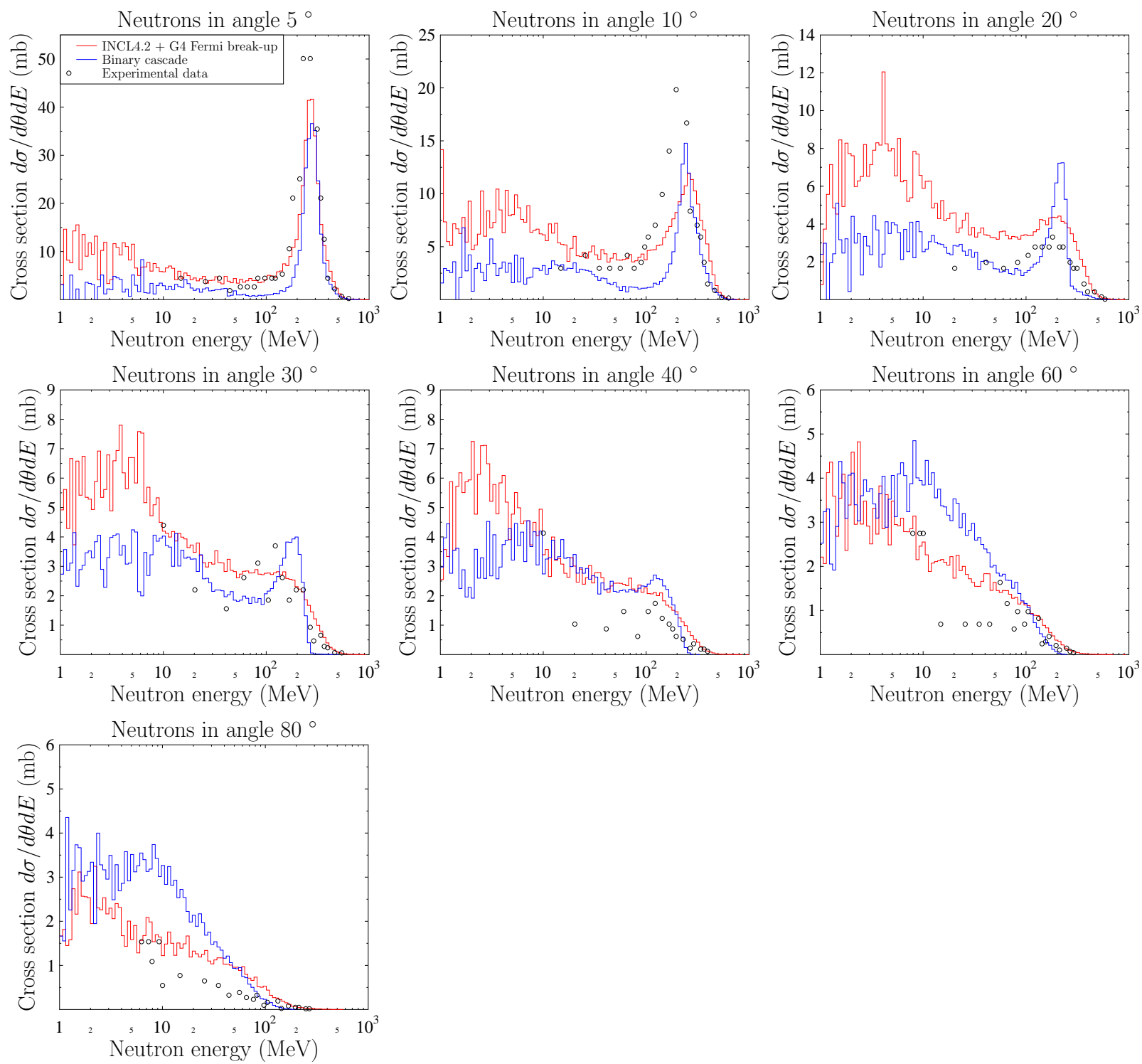

Fig. 2 Double-differential neutron energy spectra for $\mathrm{C}+\mathrm{C}$ at $290 \mathrm{MeV} /$ nucleon. INCL and Geant4 Binary cascade are compared against experimental data from Reference 13.

results of INCL4.2 (Geant4 C++ version), INCL4.3 (FORTRAN version) and Binary cascade against experimental data. The results are shown in Fig. 3. In both the experiment and calculations only fragments of oxygen and chlorine were studied. It should also be noted that in the experiment carbon was used as target but for technical reasons all calculations with the models were done using inverse kinematics (i.e. carbon as the projectile) and therefore in the calculation results we actually look at target fragmentation. The results of these comparisons show that the combination of INCL4.2 with Geant4 Fermi break-up and ABLA evaporation/fission model performs quite well when compared against Binary cascade and the FORTRAN version of INCL4.3 which uses ABLA evaporation/fission whatever the mass of the remnant.

For the first case, oxygen target, INCL4.3 (FORTRAN version) uses ABLA for de-excitation but in INCL4.2 (C++
Geant 4 version) we use a combination of ABLA and Geant4 Fermi break-up depending on the mass of the remnant produced by the cascade. In the chlorine case cascade remnants are significantly heavier than mass 16 so both versions of INCL use ABLA for de-excitation.

The oxygen and chlorine fragmentation plots in Fig. 3 show a slight disagreement between the FORTRAN version INCL4.3 and the C++ version of INCL4.2. This difference can presumably be mostly attributed to the main difference between INCL4.2 and INCL4.3: light cluster emission in the cascade stage. In the case of oxygen fragmentation both codes also use slightly different de-excitation scheme and this could affect the fragment distributions as well.

\section{Thick Water Target}

We have started doing preliminary tests using a full Geant 4 

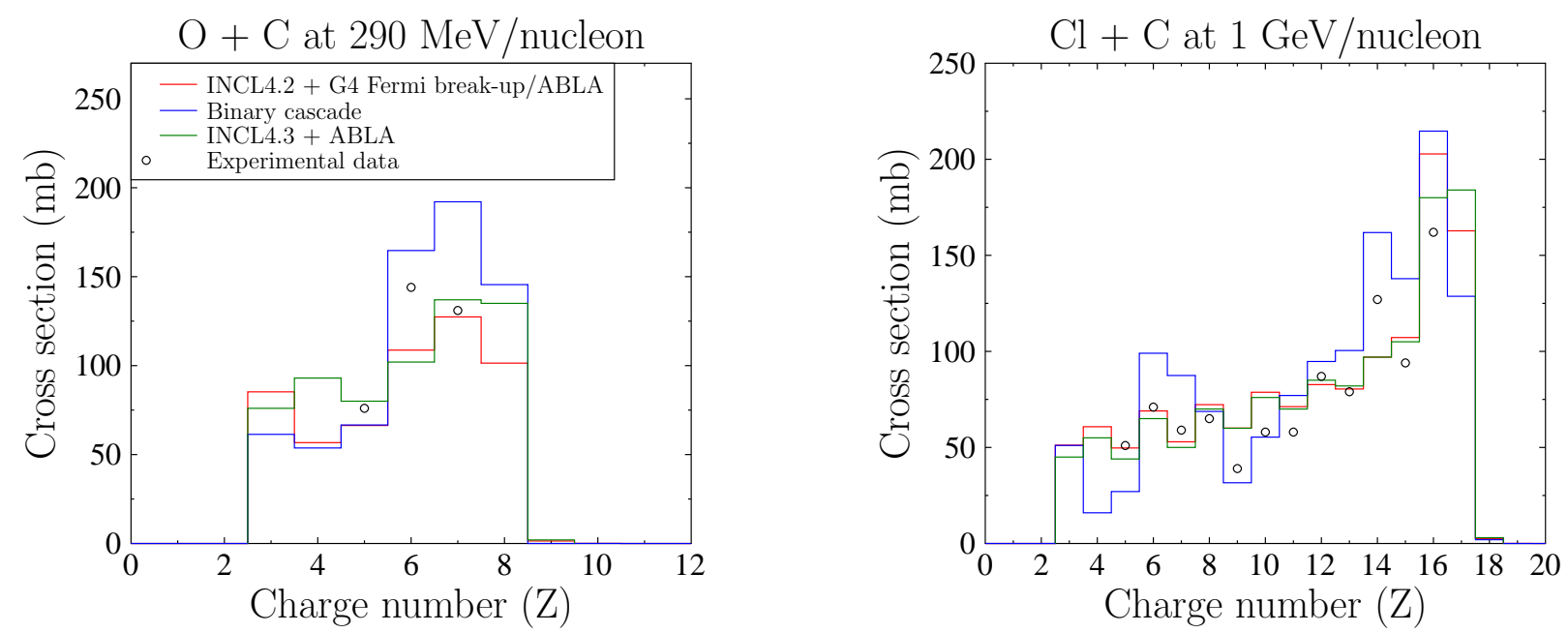

Fig. 3 Oxygen (left) and chlorine (right) fragmentation calculated with INCL4.2 (C++) coupled to Geant4 Fermi break-up for light cascade remnants (mass 16 and below) and ABLA for heavier remnants, Binary cascade, and INCL4.3 (FORTRAN) coupled to ABLA compared against experimental data from References 14,15 . For technical reasons all calculations are done using inverse kinematics.

simulation with thick water target. For this purpose we use a modified version of the Geant4 hadron therapy example application included in the standard Geant 4 distribution.

In our simulation setup we shoot a $200 \mathrm{MeV} /$ nucleon carbon beam to a $12.78 \mathrm{~cm}$ thick water target and record the outcoming particles behind the target. A preliminary neutron production comparison between INCL and Geant4 Binary cascade is shown in Fig. 4.

The physics performance of both INCL (using physics list QGSP_INCL_ABLA) and Binary cascade (using physics list QGSP_BIC_EMY) is quite good as far as neutron production is concerned.

\section{Ongoing Development: INCL Redesign in C++}

The design of the INCL FORTRAN code has remained relatively stable for more than a decade while physics performance and modeling features of the code have been significantly improved. This has made the codebase monolithic and difficult to develop further. It has become apparent that the current design has reached the end of its life. Since the Geant 4 version of INCL4.2 is basically only a minimal translation of FORTRAN code to $\mathrm{C}++$, sort of "FORTRAN in C++ syntax", it inherits all the problems of the FORTRAN version. Additionally the monolithic nature of the code makes it difficult to utilize the full potential of $\mathrm{C}++$ capabilities.

Another important problem in the current development model of INCL is that we have several "forked" versions of the code: the main line of development (version INCL4.6) and the MCNPX version (currently INCL4.2, INCL4.5 interface under development) in FORTRAN and INCL4.2 translation in $\mathrm{C}++$ for Geant 4 . Additionally the light ion extension that was first introduced in INCL4.3 has now been added to the Geant4 version of INCL and will be released in 2010. The MCNPX version of INCL is essentially the same code as the standalone version, except that it has been modified to work inside of a transport code. The redesign project gives us an opportunity to unify all these different versions under a single source tree and allows us to deliver the same physics features consistently for Geant4, MCNPX and as a standalone thin-target calculation code without duplication of effort.

The redesign project gives us an opportunity to revisit the physics ideas of INCL and experiment with features that would be very difficult to implement in current versions of INCL. Some examples of things that are difficult with the current codebase are changing the way particles are tracked or collisions are detected and implementing more realistic handling two of nuclear potentials in ion-ion collisions.

\section{Conclusions}

We have presented the INCL intra-nuclear cascade model implementation in Geant 4 and the INCL light ion projectile extension. The first results are in good agreement with experiment and physics performance of INCL is competitive with Geant4 Binary cascade.

We are now in good position to perform thick target studies and comparisons of INCL against experimental data in the case of light-ion collisions. Our first results are quite encouraging. However, there are still some open questions with regards to the physics list we should use with INCL. An especially important question is what to do for low energy collisions. In the physics list we used in this study we use INCL for nucleon, pion and ion projectiles from energy $3 \mathrm{GeV} /$ nucleon down to reaction threshold. Unfortunately some of the basic assumptions of intra-nuclear cascade start breaking down when energies fall significantly below $100 \mathrm{MeV} /$ nucleon. One available option is to investigate the use of other potentially more suitable Geant 4 models for low energy collisions and compare their results against those given by INCL. 

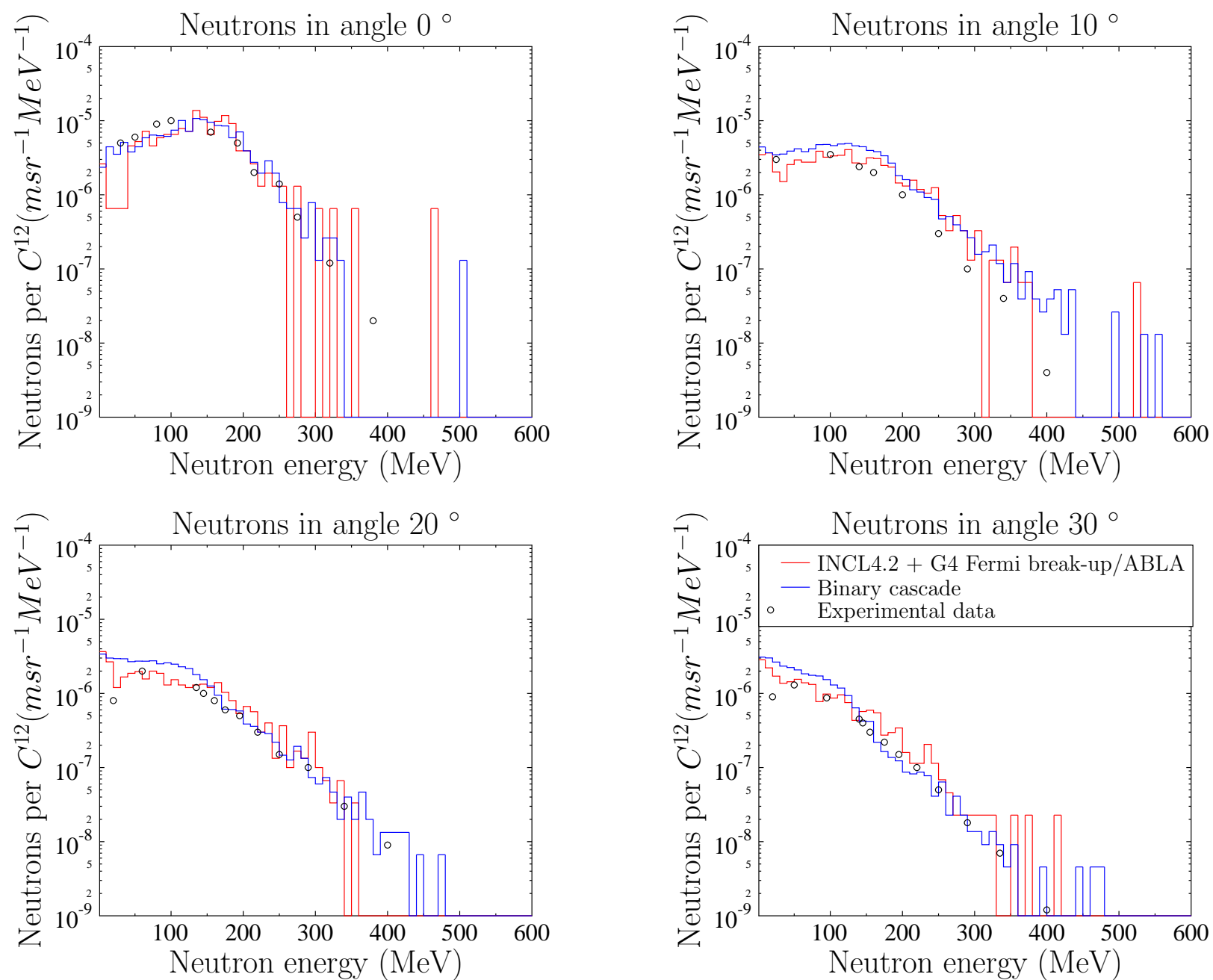

Fig. 4 Neutron double-differential energy spectra in C + water with target thickness of $12.78 \mathrm{~cm}$ at 200 $\mathrm{MeV} /$ nucleon. INCL4.2 with Geant4 Fermi break-up and Binary cascade are compared against data from Reference 16.

The validation of the light ion extension will continue and it will be included in the Geant4 9.4 release in December 2010.

\section{Acknowledgment}

We are grateful to José-Manuel Quesada and Mike Kelsey (Geant4 collaboration) for their insightful comments and suggestions for improvements concerning this paper. This work has been partly funded by Helsinki Institute of Physics and Fortum Foundation.

\section{References}

1) A. Boudard et al., "Intra-nuclear cascade model for a comprehensive description of spallation reaction data," Phys. Rev., C66, 044615 (2002).

2) J. Benlliure et al., "Calculated nuclide production yields in relativistic collisions of fissile nuclei," Nuc. Phys., A628, 458 (1998).

3) J. J. Gaimard et al., "A re-exxamination of the abrasion-ablation model for the description of the nuclear fragmentation reac- tion," Nuc. Phys., A531, 709 (1991).

4) A. R. Junghans et al., "Projectile-fragment yeields as a probe for the collective enhancement in the nuclear level density," Nuc. Phys., A629, 635 (1998).

5) Geant 4 collaboration, "Geant 4 - a simulation toolkit," Nucl. Instr. Meth. Phys. Res., A506, 250-303 (2003).

6) Laurie S. Waters, Gregg W. McKinney, Joe W. Durkee, Michael L. Fensin, John S. Hendricks, Michael R. James, Russell C. Johns, Denise B. Pelowitz, "The MCNPX Monte Carlo Radiation Transport Code," AIP Conf. Proc., 896, 81-90 (2007).

7) Denise B. Pelowitz (Ed.), MCNPX User's Manual, Version 2.6.0, LA-CP-07-1473, Los Alamos National Laboratory (LANL) (2007).

8) J. S. Hendricks et al., "MCNPX 2.6.0 Extensions," LA-UR-082216, Los Alamos National Laboratory (LANL) (2008).

9) A. Heikkinen, P. Kaitaniemi, A. Boudard, "Implementation of INCL4 cascade and ABLA evaporation codes in Geant4," $J$. Phys. Conf. Ser., 119, 032024 (2008).

10) A. Boudard, J. Cugnon, P. Kaitaniemi, S. Leray, D. Mancusi, "Simulation of light ion collisions from Intra Nuclear Cascade 
(INCL-Fermi Breakup) relevant for medical irradiations and radioprotection", Proc. Int. Topical Meeting on Nuclear Research Applications and Utilization of Accelerators 2009, Vienna, Austria, May 5-8, 2009 (2010).

11) R. E. Prael H. Liechtenstein, LA-UR-89-3014, Los Alamos National Laboratory (LANL) (1989).

12) H. Sato et al., "Measurements of double-differential neutron production cross sections by $135 \mathrm{MeV} /$ nucleon $\mathrm{He}, \mathrm{C}, \mathrm{Ne}$ and $95 \mathrm{MeV} /$ nucleon Ar ions," Phys. Rev., C64, 034607 (2001).

13) Y. Iwata et al., "Double differential cross-sections for the neutron production from heavy ion reactions at energies $\mathrm{E} / \mathrm{A}=290$ MeV to 600 MeV," Phys. Rev., C64, 054609 (2001).
14) C. Zeitlin et al., "Fragmentation of ${ }^{14} \mathrm{~N},{ }^{16} \mathrm{O},{ }^{20} \mathrm{Ne}$, and ${ }^{24} \mathrm{Mg}$ nuclei at 290 to $100 \mathrm{Me} /$ nucleon," Phys. Rev., C83, 034909 (2011).

15) C. Zeitlin et al., "Fragmentation cross sections of mediumenergy ${ }^{35} \mathrm{Cl},{ }^{40} \mathrm{Ar}$ and ${ }^{48} \mathrm{Ti}$ beams on elemental targets," Phys. Rev., C77, 034605 (2008).

16) K. Gunzert-Marx, H. Iwase, D. Schardt, R. S. Simon, "Secondary beam fragments produced by $200 \mathrm{MeV} / \mathrm{u}{ }^{12} \mathrm{C}$ ions in water and their dose contribution in radiotherapy," New J. Phys., 10075003 (2008). 